\title{
Density-functional theory with optimized effective potential and self-interaction correction for ground states and autoionizing resonances
}

\author{
Xiao-Min Tong* and Shih-I Chu \\ Department of Chemistry, University of Kansas, Lawrence, Kansas 66045
}

(Received 29 August 1996)

\begin{abstract}
We present a self-interaction-free density-functional theory (DFT) for the treatment of both the static properties of the ground states and the photoionization processes of many-electron systems. The method is based on the Krieger-Li-Iafrate (KLI) treatment of the optimized effective potential (OEP) theory and the incorporation of an explicit self-interaction correction (SIC) term. Such an extended OEP-KLI-SIC method uses only orbital-independent single-particle local potentials and is thus computationally more efficient and yet maintains good accuracy. The usefulness of the procedure is examined by the studies of the static properties of the ground states of atoms $(Z \leqslant 18)$ and the dynamical photoionization processes involving autoionizing resonances. Both the energy functionals of the local spin-density approximation (LSDA) and Becke's exchange energy functional and the correlation energy functional of Lee-Yang-Parr (BLYP) are used as the input to the KLI-SIC calculations. It is found that the implementation of the KLI-SIC procedure gives rise to optimized effective potentials that possess the correct behavior in both short-range and long-range regimes. As a consequence, the LSDA and BLYP ionization potentials are significantly improved. For higher- $Z$ atoms, the improvement of the LSDA total energies and the ionization potentials are particularly remarkable, approaching the experimental or exact values. As another test of the KLI-SIC method, we have performed the calculation of the photoionization cross sections of the $\mathrm{Ne}$ atom using both the time-independent and time-dependent LSDA (TDLSDA) methods. We found that the TDLSDA results agree closely with the experimental data in the broad peak region, followed by a series of sharp resonances due to the $2 s \rightarrow n p$ resonant transitions. The calculated linewidths and resonance line profile parameters are in reasonable agreement with both the experimental and the configuration-interaction ( $R$-matrix) results, demonstrating the usefulness of the KLI-SIC procedure for achieving accurate DFT calculations in both static properties and dynamical processes. [S1050-2947(97)05504-2]

PACS number(s): 31.15.Ew, 32.80.Fb, 32.80.Dz
\end{abstract}

\section{INTRODUCTION}

Since the fundamental work of Hohenberg and Kohn [1] and Kohn and Sham [2], the density-functional theory (DFT) has undergone significant theoretical and computational advances in recent years. DFT has become a widely used formalism for electronic-structure calculations of the groundstate properties of atoms, molecules, and solids [3-7]. In the Kohn-Sham DFT formalism [2], the electron density is decomposed into a set of orbitals, leading to a set of oneelectron Schrödinger-like equations to be solved selfconsistently. The Kohn-Sham equations are structurally similar to the Hartree-Fock equations, but include, in principle, exactly the many-body effects through a local exchange-correlation (xc) potential. Thus DFT is computational much less expensive than the traditional $a b$ initio many-electron wave-function approaches and this accounts for its great success for large systems. However, the DFT is well developed mainly for the ground-state properties. The treatment of excited states and time-dependent processes within the DFT is much less developed.

As the exact xc-energy functional is unknown, one needs to use approximations in actual computations. The simplest

\footnotetext{
*Permanent address: Institute of Physics, P.O. Box, Chinese Academy of Science, Beijing 100080, People's Republic of China.
}

approximation for the xc-energy functional is through the local-density approximation (LDA) or local spin-density approximation (LSDA) (the LDA and LSDA are equivalent for closed-shell atoms) $[3,8]$ of homogeneous electronic gas. A deficiency of the LDA (LSDA) is that the xc potential decays exponentially and does not have the correct long-range behavior. As a result, the electrons are too weakly bound and for negative ions even unbound. Further, previous LDA calculations of the photoionization of rare-gas atoms [9] did not exhibit the autoionizing resonances. More accurate explicit forms of xc-energy functionals using generalized gradient corrections are available [10-13]. However, the xc potentials derived from these explicit $\mathrm{xc}$ functionals suffer the similar problem and do not have the proper long-range Coulomb tail $-1 / r$. Thus, while the total energies of the ground states of atoms predicted by these xc density functionals [10-13] are rather accurate, the ionization potentials obtained from the highest occupied orbital energies of neutral atoms are less satisfactory [14]. For the proper treatment of photoionization and multiphoton ionization processes, it is important that both the ionization potentials and the excited-state properties be described more accurately.

In this paper we present an extension of the DFT for more accurate treatment of both the ionization potentials of the ground states as well as for the photoabsorption spectrum of autoionizing resonances. The method is based on an extension of the optimized effective potential formalism along with a self-interaction-correction procedure for various 
exchange-correlation energy functionals to be described in the next section (Sec. II). The usefulness of the proposed procedure is demonstrated by a study of the total energies and ionization potentials of atoms $(Z \leqslant 18)$ in Sec. III and the photoionization of the $\mathrm{Ne}$ atom in Sec. IV. This is followed by a conclusion in Sec. V.

\section{THE OPTIMIZED EFFECTIVE POTENTIAL METHOD WITH SELF-INTERACTION CORRECTION}

In the Kohn-Sham (KS) DFT formulation [2], one solves the following set of one-electron Schrödinger-like equation for $N$-electron systems (in atomic units):

$$
\begin{array}{r}
\hat{H}_{\mathrm{KS}} \psi_{i \sigma}(\mathbf{r})=\left[-\frac{1}{2} \nabla^{2}+v_{\mathrm{eff}, \sigma}(\mathbf{r})\right] \psi_{i \sigma}(\mathbf{r})=\varepsilon_{i \sigma} \psi_{i \sigma}(\mathbf{r}), \\
i=1,2, \ldots, N_{\sigma},
\end{array}
$$

where $v_{\text {eff, } \sigma}(\mathbf{r})$ is the effective KS potential and $\sigma$ is the spin index. The total density is given by

$$
\begin{aligned}
\rho(\mathbf{r}) & =\sum_{\sigma} \sum_{i=1}^{N_{\sigma}}\left|\psi_{i \sigma}(\mathbf{r})\right|^{2}=\sum_{i=1}^{N_{\uparrow}}\left|\psi_{i \uparrow}(\mathbf{r})\right|^{2}+\sum_{i=1}^{N_{\downarrow}}\left|\psi_{i \downarrow}(\mathbf{r})\right|^{2} \\
& =\rho_{\uparrow}(\mathbf{r})+\rho_{\downarrow}(\mathbf{r})
\end{aligned}
$$

and the ground-state wave function is determined by

$$
\Psi=\frac{1}{\sqrt{N !}} \operatorname{det}\left[\psi_{1} \psi_{2} \cdots \psi_{N}\right]
$$

The total energy of the ground state is obtained by the minimization of the Hohenberg-Kohn energy functional $[1,3]$

$$
E\left[\rho_{\uparrow}, \rho_{\downarrow}\right]=T_{s}[\rho]+J[\rho]+E_{\mathrm{xc}}\left[\rho_{\uparrow}, \rho_{\downarrow}\right]+\int v_{\mathrm{ext}}(\mathbf{r}) \rho(\mathbf{r}) d^{3} \mathbf{r} .
$$

Here $T_{s}$ is the noninteracting KS kinetic energy,

$$
T_{s}=\sum_{\sigma} \sum_{i=1}^{N_{\sigma}}\left\langle\psi_{i \sigma}\left|-\frac{1}{2} \nabla^{2}\right| \psi_{i \sigma}\right\rangle
$$

$v_{\text {ext }}(\mathbf{r})$ is the "external" potential due to the electronnucleus interaction, $J[\rho]$ is the classical electron-electron repulsive energy

$$
J[\rho]=\frac{1}{2} \iint \frac{\rho(\mathbf{r}) \rho\left(\mathbf{r}^{\prime}\right)}{\left|\mathbf{r}-\mathbf{r}^{\prime}\right|} d^{3} \mathbf{r} d^{3} \mathbf{r}^{\prime},
$$

and $E_{\mathrm{xc}}\left[\rho_{\uparrow}, \rho_{\downarrow}\right]$ is the exchange-correlation energy. Minimization of the total-energy functional (4) subject to the constraint

$$
\int \rho_{\sigma}(\mathbf{r}) d^{3} \mathbf{r}=N_{\sigma}
$$

gives rise to the KS equations (1) with the effective potential

$$
\begin{aligned}
v_{\mathrm{eff}, \sigma}(\mathbf{r})= & v_{\mathrm{ext}}(\mathbf{r})+\frac{\delta J[\rho]}{\delta \rho_{\sigma}(\mathbf{r})}+\frac{\delta E_{\mathrm{xc}}\left[\rho_{\uparrow}, \rho_{\downarrow}\right]}{\delta \rho_{\sigma}(\mathbf{r})}=v_{\mathrm{ext}}(\mathbf{r}) \\
& +\int \frac{\rho\left(\mathbf{r}^{\prime}\right)}{\left|\mathbf{r}-\mathbf{r}^{\prime}\right|} d^{3} \mathbf{r}^{\prime}+v_{\mathrm{xc}, \sigma}(\mathbf{r})
\end{aligned}
$$

where $v_{\mathrm{xc}, \sigma}(\mathbf{r})$ is the exchange-correlation potential

$$
v_{\mathrm{xc}, \sigma}(\mathbf{r})=\frac{\delta E_{\mathrm{xc}}\left[\rho_{\uparrow}, \rho_{\downarrow}\right]}{\delta \rho_{\sigma}(\mathbf{r})}
$$

The KS equations are to be solved self-consistently, starting from some initial estimate of the density $\rho_{\sigma}(\mathbf{r})$, until convergence is reached. In actual calculations, the KS Hamiltonian in Eq. (1) must be fixed by a particular choice of the xc energy functional $E_{\mathrm{xc}}\left[\rho_{\uparrow}, \rho_{\downarrow}\right]$. However, most $E_{\mathrm{xc}}\left[\rho_{\uparrow}, \rho_{\downarrow}\right]$ forms, such as the LSDA form as well as the more recent ones using gradient corrections [10-13], contain spurious self-interaction contributions. Such a self-interaction contribution can be seen from Eq. (4), where the last two terms $J[\rho]$ and $E_{\mathrm{xc}}\left[\rho_{\uparrow}, \rho_{\downarrow}\right]$ should, in principle, cancel each other exactly in the limit of one-electron system, if the exact form for $E_{\mathrm{xc}}\left[\rho_{\uparrow}, \rho_{]}\right]$is used. In practice, $E_{\mathrm{xc}}\left[\rho_{\uparrow}, \rho_{\downarrow}\right]$ needs to be approximated, leading to the self-interaction energy. The existence of such self-interaction energy is the main source of error responsible for the incorrect long-range behavior of the exchange-correlation potential $v_{\mathrm{xc}, \sigma}(\mathbf{r})$. Thus the elimination of the self-interaction contribution is essential for the proper treatment of the ionization potentials and excited-state properties.

Various approaches have been suggested to remove the self-interaction problem [3]. Perdew and Zunger [15] proposed the self-interaction correction (SIC) version of a given approximate exchange-correlation energy functional $E_{\mathrm{xc}}\left[\rho_{\uparrow}, \rho_{\downarrow}\right]$,

$$
E_{\mathrm{xc}}^{\mathrm{SIC}}\left[\rho_{\uparrow}, \rho_{\downarrow}\right]=E_{\mathrm{xc}}\left[\rho_{\uparrow}, \rho_{\downarrow}\right]-\sum_{\sigma} \sum_{i=1}^{N_{\sigma}}\left\{J\left[\rho_{i \sigma}\right]+E_{\mathrm{xc}}\left[\rho_{i \sigma}, 0\right]\right\},
$$

where $\rho_{i \sigma}$ is the single-particle density of the $i$ th KS spin orbital. In the limit that exact $E_{\mathrm{xc}}\left[\rho_{\uparrow}, \rho_{\downarrow}\right]$ is used, the SIC term $\left\{J\left[\rho_{i \sigma}\right]+E_{\mathrm{xc}}\left[\rho_{i \sigma}, 0\right]\right\}$ vanishes. Thus this SIC correction term can be also considered as a measure of the deviation of a given approximate $E_{\mathrm{xc}}\left[\rho_{\uparrow}, \rho_{\downarrow}\right]$ from the exact result. The use of the SIC energy functional $E_{\mathrm{xc}}^{\mathrm{SIC}}\left[\rho_{\uparrow}, \rho_{\downarrow}\right]$ [Eq. (10)], however, leads to different potentials for different orbitals. Such an orbital dependence of the one-electron potentials causes the orbitals to be nonorthogonal. Additional effort must be taken to achieve orthogonal SIC spin orbitals.

Another promising approach for improving $E_{x c}\left[\rho_{\uparrow}, \rho_{l}\right]$ is provided by the so-called optimized effective potential (OEP) method $[16,17]$. In this approach, one solves a set of oneelectron equations, similar to the KS equations in Eq. (1), 


$$
\begin{array}{r}
\hat{H}_{\mathrm{OEP}} \phi_{i \sigma}(\mathbf{r})=\left[-\frac{1}{2} \nabla^{2}+V_{\sigma}^{\mathrm{OEP}}(\mathbf{r})\right] \phi_{i \sigma}(\mathbf{r})=\varepsilon_{i \sigma} \phi_{i \sigma}(\mathbf{r}), \\
i=1,2, \ldots, N_{\sigma} .
\end{array}
$$

The optimized effective potential $V_{\sigma}^{\mathrm{OEP}}(\mathbf{r})$ is obtained by the requirement that the spin orbitals $\left\{\phi_{i \sigma}\right\}$ in Eq. (11) are those that minimize the total-energy functional $E\left[\left\{\phi_{i \uparrow}, \phi_{j \downarrow}\right\}\right]$,

$$
\frac{\delta E^{\mathrm{OEP}}\left[\left\{\phi_{i \uparrow}, \phi_{j \downarrow}\right\}\right]}{\delta V_{\sigma}^{\mathrm{OEP}}(\mathbf{r})}=0,
$$

where

$$
\begin{aligned}
E^{\mathrm{OEP}}\left[\left\{\phi_{i \uparrow}, \phi_{j \downarrow}\right\}\right]= & T_{s}\left[\left\{\phi_{i \uparrow}, \phi_{j \downarrow}\right\}\right]+J\left[\left\{\phi_{i \uparrow}, \phi_{j \downarrow}\right\}\right] \\
& +E_{\mathrm{xc}}\left[\left\{\phi_{i \uparrow}, \phi_{j \downarrow}\right\}\right]+\int v_{\mathrm{ext}}(\mathbf{r}) \rho(\mathbf{r}) d^{3} \mathbf{r} .
\end{aligned}
$$

Equation (13) for the energy functional $E^{\mathrm{OEP}}\left[\left\{\phi_{i \uparrow}, \phi_{j \downarrow}\right\}\right]$ has the same form as that of Hohenberg-Kohn energy functional given in Eq. (4), since the total energy is a functional of the density $\rho$, and the density is determined by the spin orbitals $\left\{\phi_{i \uparrow}, \phi_{j \downarrow}\right\}$.

Equation (12) can be written as, using the chain rule for functional derivative,

$$
\sum_{j} \int d^{3} \mathbf{r}^{\prime} \frac{\delta E^{\mathrm{OEP}}\left[\left\{\phi_{i \uparrow,} \phi_{i^{\prime} \downarrow}\right\}\right]}{\delta \phi_{j \sigma}\left(\mathbf{r}^{\prime}\right)} \cdot \frac{\delta \phi_{j \sigma}\left(\mathbf{r}^{\prime}\right)}{\delta V_{\sigma}^{\mathrm{OEP}}(\mathbf{r})}+\text { c.c. }=0 .
$$

While the physical idea of the OEP method is simple and appealing, Eq. (14) leads to an integral equation that is computionally impractical to solve. Recently, Krieger, $\mathrm{Li}$, and Iafrate [18-22] have worked out an approximate, albeit accurate, procedure to circumvent this difficulty, reducing the determination of $V_{\sigma}^{\mathrm{OEP}}$ to the solution of simple linear equations. As will be shown below, the Krieger-Li-Iafrate (KLI) method provides also a convenient procedure to obtain orbital-independent optimized effective potential that is selfinteraction-free. The KLI method has been used mainly in the exchange-only limit [20-22] and the results are nearly identical to those obtained from the exact OEP method. More recently, Grabo and Gross [14] have further employed the KLI method to include the correlation energy functional and found that both the total energies and the ionization potentials of neutral atoms can be well represented.

Encouraged by these recent advancements, we present below an extension of the KLI procedure to explicitly incorporate the SIC term given in Eq. (10). The motivations of such a development are as follows. (a) In all the KLI calculations so far [14,20-22], the exchange part of the density functional contains a Hartree-Fock-like nonlocal potential. While such a procedure provides accurate results for the exchange part of $E_{\mathrm{xc}}$, it is computationally more expensive than the traditional DFT calculations where only local single-particle potential are used. Thus we are interested in exploring an approximate and yet accurate procedure within the KLI framework involving only the use of local potentials. This would greatly speed up the computations of the static and dynamical properties of many-electron systems. Such a simplified procedure is particularly desirable when the timedependent processes are considered [23]. In the latter case, one needs to reconstruct the optimized effective potential for each small time step. (b) As will be shown below, the proposed KLI-SIC procedure, similar to the original KLI method, allows also the construction of a self-interactionfree effective potential that is orbital independent. This avoids the problems associated with the conventional SIC procedure [15] discussed earlier. Further, the optimized effective potential so constructed, denoted by $V_{\mathrm{KLI}, \sigma}^{\mathrm{SIC}}(\mathbf{r})$ below, has the proper long-range $(-1 / r)$ behavior and thus is suitable for the determination of both ground- and excited-state properties of many-electron systems. (Recently, we became aware that a similar KLI-SIC procedure has been used by Krieger and his co-workers [24,25] in conjunction with the LSDA energy functional. In our present work, in addition to the LSDA energy functional, we have also considered other more refined exchanged-correlation energy functionals.) We now outline the key equations in the extended procedure.

Define the total energy functional with SIC to be [26]

$$
\begin{aligned}
E_{\mathrm{SIC}}^{\mathrm{OEP}}\left[\left\{\phi_{i \uparrow}, \phi_{j \downarrow}\right\}\right]= & E^{\mathrm{OEP}}\left[\left\{\phi_{i \uparrow}, \phi_{j \downarrow}\right\}\right]-\sum_{\sigma} \sum_{i}\left\{J\left[\rho_{i \sigma}\right]\right. \\
& \left.+E_{\mathrm{xc}}\left[\rho_{i \sigma}, 0\right]\right\},
\end{aligned}
$$

where $E^{\mathrm{OEP}}\left[\left\{\phi_{i \uparrow}, \phi_{j \downarrow}\right\}\right]$ is given in Eq. (13). Following the OEP-KLI procedure, one finds that

$$
\begin{aligned}
V_{\mathrm{SIC}, \sigma}^{\mathrm{OEP}}(\mathbf{r})= & v_{\mathrm{ext}}(\mathbf{r})+\int \frac{\rho\left(\mathbf{r}^{\prime}\right)}{\left|\mathbf{r}-\mathbf{r}^{\prime}\right|} d^{3} \mathbf{r}^{\prime}+\frac{\delta E_{\mathrm{xc}}\left[\rho_{\uparrow} \rho_{\downarrow}\right]}{\delta \rho_{\sigma}(\mathbf{r})} \\
& +V_{\mathrm{SIC}, \sigma}(\mathbf{r}),
\end{aligned}
$$

where

$$
\begin{gathered}
V_{\mathrm{SIC}, \sigma}(\mathbf{r})=\sum_{i} \frac{\rho_{i \sigma}(\mathbf{r})}{\rho_{\sigma}(\mathbf{r})}\left\{v_{i \sigma}(\mathbf{r})+\left[\bar{V}_{\mathrm{SIC}, \sigma}^{i}-\bar{v}_{i \sigma}\right]\right\}, \\
v_{i \sigma}(\mathbf{r})=-\int \frac{\rho_{i \sigma}\left(\mathbf{r}^{\prime}\right)}{\left|\mathbf{r}-\mathbf{r}^{\prime}\right|} d^{3} \mathbf{r}^{\prime}-\frac{\delta E_{\mathrm{xc}}\left[\rho_{i \sigma}, 0\right]}{\delta \rho_{i \sigma}(\mathbf{r})}
\end{gathered}
$$

and

$$
\begin{aligned}
\bar{V}_{\mathrm{SIC}, \sigma}^{i} & =\left\langle\phi_{i \sigma}\left|V_{\mathrm{SIC}, \sigma}(\mathbf{r})\right| \phi_{i \sigma}\right\rangle, \\
\bar{v}_{i \sigma} & =\left\langle\phi_{i \sigma}\left|v_{i \sigma}(\mathbf{r})\right| \phi_{i \sigma}\right\rangle .
\end{aligned}
$$

In Eq. (17), the last two terms $\bar{V}_{\mathrm{SIC}, \sigma}^{i}$ and $\bar{v}_{i \sigma}$ are constants, though the value of $\bar{V}_{\text {SIC, } \sigma}^{i}$ is unknown. The KLI method suggests a way to calculate $\left(\bar{V}_{\text {SIC, } \sigma}^{i}-\bar{v}_{i \sigma}\right)$ through a solution of the linear equations

$$
\begin{array}{r}
\sum_{i=1}^{N_{\sigma}-1}\left(\delta_{j i, \sigma}-M_{j i, \sigma}\right)\left(\bar{V}_{\mathrm{SIC}, \sigma}^{i}-\bar{v}_{i \sigma}\right)=\bar{V}_{j \sigma}^{s}-\bar{v}_{j \sigma}, \\
j=1,2, \ldots, N_{\sigma}-1,
\end{array}
$$

where 
TABLE I. Total energies (in a.u.) of ground states of neutral atoms $(Z \leqslant 18)$ calculated by various exchange-correlation energy functionals.

\begin{tabular}{lccccccc}
\hline \hline & \multicolumn{2}{c}{ Non-KLI-SIC } & \multicolumn{2}{c}{ KLI-SIC } & \multicolumn{2}{c}{ KLI } & \\
Atom & HF $^{\mathrm{a}}$ & LSDA & BLYP & LSDA & BLYP & GG $^{\mathrm{b}}$ & Exact $^{\mathrm{c}}$ \\
\hline $\mathrm{He}$ & -2.862 & -2.724 & -2.907 & -2.862 & -2.905 & -2.903 & -2.904 \\
$\mathrm{Li}$ & -7.433 & -7.193 & -7.483 & -7.434 & -7.481 & -7.483 & -7.478 \\
$\mathrm{Be}$ & -14.573 & -14.223 & -14.663 & -14.578 & -14.651 & -14.665 & -14.667 \\
$\mathrm{~B}$ & -24.529 & -24.064 & -24.647 & -24.549 & -24.634 & -24.656 & -24.654 \\
$\mathrm{C}$ & -37.689 & -37.112 & -37.845 & -37.745 & -37.832 & -37.849 & -37.845 \\
$\mathrm{~N}$ & -54.401 & -53.709 & -54.595 & -54.506 & -54.584 & -54.591 & -54.589 \\
$\mathrm{O}$ & -74.810 & -73.992 & -75.081 & -74.962 & -75.069 & -75.072 & -75.067 \\
$\mathrm{~F}$ & -99.409 & -98.474 & -99.761 & -99.635 & -99.749 & -99.730 & -99.734 \\
$\mathrm{Ne}$ & -128.547 & -127.491 & -128.976 & -128.859 & -128.966 & -128.920 & -128.939 \\
$\mathrm{Na}$ & -161.859 & -160.644 & -162.297 & -162.217 & -162.290 & -162.256 & -162.257 \\
$\mathrm{Mg}$ & -199.615 & -198.249 & -200.097 & -200.027 & -200.080 & -200.062 & -200.059 \\
$\mathrm{Al}$ & -241.877 & -240.356 & -242.385 & -242.341 & -242.354 & -242.362 & -242.356 \\
$\mathrm{Si}$ & -288.854 & -287.182 & -289.394 & -289.378 & -289.340 & -289.375 & -289.374 \\
$\mathrm{P}$ & -340.719 & -338.889 & -341.284 & -341.299 & -341.202 & -341.272 & -341.272 \\
$\mathrm{~S}$ & -397.505 & -395.519 & -398.134 & -398.148 & -398.018 & -398.128 & -398.139 \\
$\mathrm{Cl}$ & -459.482 & -457.344 & -460.172 & -460.197 & -460.016 & -460.164 & -460.196 \\
$\mathrm{Ar}$ & -526.818 & -524.518 & -527.558 & -527.599 & -527.358 & -527.553 & -527.604 \\
\hline \hline
\end{tabular}

${ }^{\mathrm{a}}$ Reference [30].

${ }^{\mathrm{b}}$ Reference [14].

${ }^{\mathrm{c}}$ The data for $Z \leqslant 10$ are from Ref. [28] and data for $Z \geqslant 11$ are from Ref. [29].

$$
M_{j i, \sigma}=\int \frac{\rho_{j \sigma}(\mathbf{r}) \rho_{i \sigma}(\mathbf{r})}{\rho_{\sigma}(\mathbf{r})} d^{3} \mathbf{r}
$$

and

$$
\bar{V}_{i \sigma}^{s}=\left\langle\phi_{i \sigma}\left|\sum_{j=1}^{N_{\sigma}} \frac{\rho_{j \sigma}(\mathbf{r}) \cdot v_{j \sigma}(\mathbf{r})}{\rho_{\sigma}(\mathbf{r})}\right| \phi_{i \sigma}\right\rangle .
$$

In an actual computation, we found that the use of Eq. (21) for the solution of $\bar{V}_{\text {SIC, } \sigma}^{i}$ may be avoided. Since the set of OEP-KLI equations in Eq. (11), with $V_{\sigma}^{\mathrm{OEP}}(\mathbf{r})$ replaced by $V_{\mathrm{SIC}, \sigma}^{\mathrm{OEP}}(\mathbf{r})$, is to be solved self-consistently along with Eq. (16), it is sufficient to use the value of $\bar{V}_{\text {SIC, } \sigma}^{i}$ from the previous iteration in Eq. (17) without needing to calculate $M_{i j, \sigma}$ and $\bar{V}_{i \sigma}^{s}$. This simplified procedure leads to the same final converged results. Finally, we choose $\bar{V}_{\text {SIC, } \sigma}^{i=N_{\sigma}}=\bar{v}_{N \sigma}$ for the highest occupied orbital as suggested by the KLI procedure. The energy of the highest occupied orbital provides an approximate value for the first ionization potential as proved by Perdew et al. [27].

\section{CALCULATION OF TOTAL ENERGIES AND IONIZATION POTENTIALS OF NEUTRAL ATOMS AND NEGATIVE IONS: THE KLI-SIC METHOD}

In this section we present the calculated results for the total ground-state energies and ionization potentials of neutral atoms and some negative ions $(Z \leqslant 18)$ using the KLISIC procedure discussed in the preceding section. For comparison, we also performed calculations of the Kohn-Sham total energies and ionization potentials without the use of the KLI-SIC. Several xc energy functionals were used, but only the results of the following two energy functionals are presented, which illustrated the main features of the KLI-SIC procedure. (a) First is the $X_{\alpha}$ exchange functional with $\alpha=2 / 3$, referred to as the LSDA in this section and Sec. IV:

$$
V_{\mathrm{xc}, \sigma}^{\mathrm{LSDA}}(\mathbf{r})=\frac{\delta E_{\mathrm{xc}}\left[\rho_{\uparrow} \rho_{\downarrow}\right]}{\delta \rho_{\sigma}(\mathbf{r})}=-\frac{3}{2} \alpha\left(\frac{6}{\pi} \rho_{\sigma}(\mathbf{r})\right)^{1 / 3} .
$$

The LSDA form is the simplest energy functional form and is the leading term of more sophisticated xc energy functionals $[10,11]$. (b) Then there is the exchange energy functional of Becke [10] and the correlation energy functional of Lee, Yang, and Parr [11], which is referred to as BLYP xc energy functional. This is one of the most commonly used functionals including the gradient corrections. Within the KLI-SIC procedure, we can define the exchange energy as

$$
E_{x}^{\mathrm{SIC}}=E_{x}\left[\rho_{\uparrow}, \rho_{\downarrow}\right]-\sum_{\sigma} \sum_{i=1}^{N_{\sigma}}\left\{J\left[\rho_{i \sigma}\right]+E_{x}\left[\rho_{i \sigma}, 0\right]\right\}
$$

and the correlation energy as

$$
E_{c}^{\mathrm{SIC}}=E_{c}\left[\rho_{\uparrow}, \rho_{\downarrow}\right]-\sum_{\sigma} \sum_{i=1}^{N_{\sigma}}\left\{E_{c}\left[\rho_{i \sigma}, 0\right]\right\} .
$$

\section{A. Total ground-state energies of neutral atoms with $Z \leqslant 18$}

In Table I we listed the calculated total energies of neutral atoms $(Z \leqslant 18)$ using various methods. The results both with and without the KLI-SIC procedure are presented. Also shown in Table I are the exact data [28,29] and the recent calculated results of Grabo and Gross [14]. In Ref. [14] the calculations were performed using also the KLI procedure, 

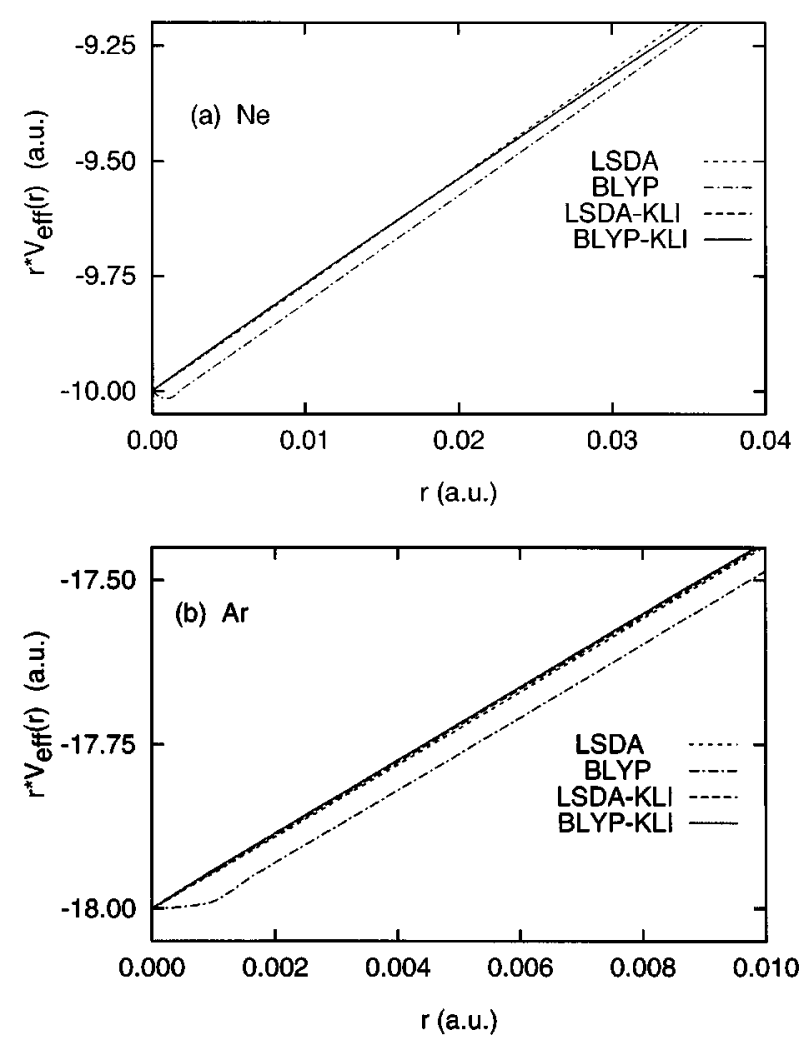

FIG. 1. Short-range one-electron effective potentials $r V_{\text {eff }}(r)$ of the LSDA and BLYP with and without the KLI-SIC near the nucleus for (a) a Ne atom and (b) an Ar atom. Note that except the BLYP potential, all the other (LSDA, LSDA-KLI-SIC, and BLYP-KLI-SIC) potentials have the proper behavior near the origin.

but the exchange part was carried out using the exact nonlocal Hartree-Fock (HF) like potentials and the correlation part performed using the Colle-Salvetti functional [31,32]. Thus it is expected that the exchange energies will be more accurately represented by this method [14]. However, the present KLI-SIC procedure uses completely local potentials and is computationally much less expensive.

Table I shows that the LSDA (without the KLI-SIC) severely underestimates the total energies and the discrepancy with the exact values increases with the atomic number $Z$. With the implementation of the KLI-SIC procedure, the LSDA shows a dramatic improvement of the total energies, particularly for higher- $Z$ atoms. In fact, for larger- $Z$ atoms, the LSDA-KLI-SIC results are considerably better than those of the HF method and surprisingly also are somewhat better than those of the BLYP with KLI-SIC. Finally, we note that our LSDA-KLI-SIC results are essentially identical to the recent calculation by Chen et al. [25], who employed a similar procedure.

The total energies of the BLYP (without the KLI SIC) are in very good agreement with the exact values. The implementation of the the KLI-SIC procedure in general does not show improvement over those without the KLI-SIC. This seems somewhat contradictory to the expectation that a better xc-energy functional should give better results. To explore the reasons responsible for such an unexpected behavior, we plot the effective one-particle potentials $V_{\text {eff }}(r)$ [Eq.
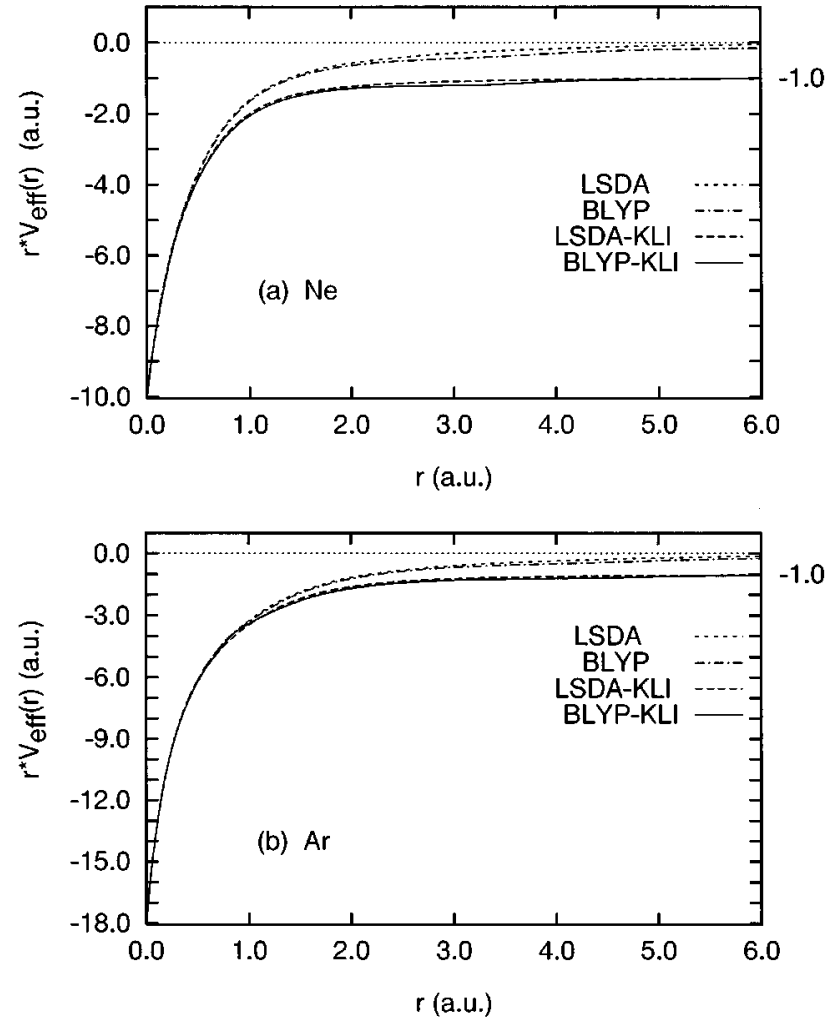

FIG. 2. One-electron effective potentials $r V_{\text {eff }}(r)$ of the LSDA and BLYP with and without the KLI SIC in a larger scale for (a) a $\mathrm{Ne}$ atom and (b) an Ar atom. Note that with the KLI-SIC, both LSDA and BLYP potentials have the correct long-range asymptotic behavior, namely, $r V_{\text {eff }}(r) \rightarrow-1$.

(8)] in Figs. 1 and 2. Figures 1(a) and 1(b) show the shortrange behavior of $r V_{\mathrm{eff}}(r)$ near the origin for $\mathrm{Ne}$ and $\mathrm{Ar}$, respectively. As can be seen, the BLYP short-range potential shows some deviation from the exact result and an abrupt change near the origin. This may be attributed to the large density derivative of the Becke exchange energy functional [10]. After the implementation of the KLI-SIC procedure, the irregular short-range behavior of the BLYP (Becke) potential is corrected as shown in Figs. 1(a) and 1(b). This explains the difference of the results of the BLYP with and without the KLI-SIC. However, since the BLYP (and Becke) energy functional expression (without the KLI SIC) contains semiempirical parameters that fit the total energies to the exact values, the BLYP total energies (after the KLI-SIC) show an "overcorrection," resulting in seemingly contradictory behavior. In reality, the BLYP effective one-particle potential after the KLI-SIC is much improved in both the shortrange (Fig. 1) and the long-range (Fig. 2) regimes. This leads to marked improvement of the BLYP ionization potentials (to be discussed below). Finally, we note that the deviation of the BLYP (Becke) short-range potential from the exact result increases with the nuclear charge $Z$, as can be clearly seen in Fig. 1 (Ne vs Ar). As a consequence, the difference of the BLYP total energies (Table I) [and exchange energies (Table II)] before and after the KLI-SIC increases with increasing $Z$.

To further understand the KLI-SIC results, we present in Table II the exchange energies calculated by various meth- 
TABLE II. Exchange energies (in a.u.) of ground states of neutral atoms $(Z \leqslant 18)$ calculated by various exchange-correlation energy functionals.

\begin{tabular}{lcccccc}
\hline \hline & \multicolumn{2}{c}{ Non-KLI-SIC } & \multicolumn{2}{c}{ KLI SIC } & \multicolumn{2}{c}{ KLI } \\
Atom & LSDA & BLYP & LSDA & BLYP & GG $^{\mathrm{a}}$ & $\mathrm{HF}^{\mathrm{b}}$ \\
\hline $\mathrm{He}$ & -0.853 & -1.019 & -1.026 & -1.028 & -1.028 & -1.026 \\
$\mathrm{Li}$ & -1.505 & -1.772 & -1.777 & -1.769 & -1.784 & -1.781 \\
$\mathrm{Be}$ & -2.278 & -2.659 & -2.658 & -2.633 & -2.674 & -2.667 \\
$\mathrm{~B}$ & -3.230 & -3.728 & -3.749 & -3.701 & -3.760 & -3.744 \\
$\mathrm{C}$ & -4.412 & -5.029 & -5.089 & -5.007 & -5.064 & -5.045 \\
$\mathrm{~N}$ & -5.837 & -6.580 & -6.691 & -6.564 & -6.610 & -6.596 \\
$\mathrm{O}$ & -7.276 & -8.156 & -8.314 & -8.143 & -8.200 & -8.175 \\
$\mathrm{~F}$ & -8.972 & -9.992 & -10.212 & -9.984 & -10.025 & -10.00 \\
$\mathrm{Ne}$ & -10.937 & -12.102 & -12.398 & -12.102 & -12.110 & -12.11 \\
$\mathrm{Na}$ & -12.702 & -14.009 & -14.355 & -13.992 & -14.017 & -14.02 \\
$\mathrm{Mg}$ & -14.535 & -15.990 & -16.383 & -15.945 & -15.997 & -15.99 \\
$\mathrm{Al}$ & -16.455 & -18.057 & -18.504 & -17.983 & -18.081 & -18.07 \\
$\mathrm{Si}$ & -18.513 & -20.264 & -20.768 & -20.154 & -20.295 & -20.28 \\
$\mathrm{P}$ & -20.711 & -22.614 & -23.177 & -22.463 & -22.649 & -22.64 \\
$\mathrm{~S}$ & -22.915 & -24.971 & -25.597 & -24.777 & -25.021 & -25.00 \\
$\mathrm{Cl}$ & -25.270 & -27.481 & -28.173 & -27.237 & -27.530 & -27.51 \\
$\mathrm{Ar}$ & -27.775 & -30.144 & -30.905 & -29.845 & -30.192 & -30.19 \\
\hline \hline
\end{tabular}

${ }^{\mathrm{a}}$ Reference [14].

${ }^{\mathrm{b}}$ Reference [33].

ods. The HF results can be considered as the "exact" values for the exchange energies. The LSDA results (without the KLI-SIC) are significantly smaller than the HF values. However, the LSDA exchange energies are markedly improved after the KLI-SIC. This is mainly due to the fact that the long-range potential is now implemented into the LSDA; see Figs. 2(a) and 2(b). For the BLYP, the exchange energies before the KLI-SIC are quite close to the HF values mainly because the Becke exchange functional [10] was designed to fit the HF limit. After the KLI-SIC, the BLYP exchange energies, although still in good agreement with the exact values, show some deviation from the HF values for larger$Z$ atoms. This is due to the problem of Becke's energy functional near the nucleus as discussed above. The exchange and total energies of Ref. [14] show closer agreement with the exact values since the exchange part was performed using nonlocal Hartree-Fock-like potentials as indicated before. In Table III we list the calculated values of BLYP correlation energies with and without the KLI-SIC. The overall agreement with the exact values and the KLI results of Ref. $[14]$ is quite good. Except for the largest- $Z$ atoms, the BLYP results with the KLI-SIC are generally slightly better than those without the KLI-SIC.

Also shown in Table III are the SIC energies calculated from the expression $\Delta E_{\mathrm{SIC}}=-\Sigma_{\sigma} \Sigma_{i}\left\{J\left[\rho_{i \sigma}\right]+E_{\mathrm{xc}}\left[\rho_{i \sigma}, 0\right]\right\}$. This SIC term is mainly responsible for the difference of the total energies of the LSDA (and BLYP) calculations with and without the KLI-SIC. In the limit that exact xc energy functionals are used, the SIC term should vanish. Thus the magnitude of the SIC energy reflects the deviation of a given xc energy functional from the exact result. Table III shows that the SIC energies for the LSDA are much larger than those of the BLYP, reflecting the well-known fact that the BLYP form is a more refined functional. However, our re-
TABLE III. Correlation energies (in a.u.) of neutral atoms $(Z \leqslant 18)$ calculated by various exchange-correlation energy functionals. Also shown are the SIC energies (in a.u.).

\begin{tabular}{lcccccc}
\hline \hline & \multicolumn{3}{c}{ Correlation energy $^{2}$} & \multicolumn{2}{c}{$\Delta E_{\text {SIC }}$} \\
Atom & BLYP $^{\mathrm{a}}$ & BLYP $^{\mathrm{b}}$ & $\mathrm{GG}^{\mathrm{c}}$ & Exact $^{\mathrm{d}}$ & KLI-LSDA & KLI-BLYP \\
\hline $\mathrm{He}$ & -0.044 & -0.044 & -0.042 & -0.042 & -0.142 & 0.000 \\
$\mathrm{Li}$ & -0.054 & -0.053 & -0.051 & -0.046 & -0.244 & 0.000 \\
$\mathrm{Be}$ & -0.096 & -0.095 & -0.093 & -0.094 & -0.357 & 0.009 \\
$\mathrm{~B}$ & -0.129 & -0.128 & -0.129 & -0.125 & -0.488 & 0.011 \\
$\mathrm{C}$ & -0.162 & -0.161 & -0.161 & -0.157 & -0.636 & 0.010 \\
$\mathrm{~N}$ & -0.193 & -0.192 & -0.188 & -0.189 & -0.801 & 0.008 \\
$\mathrm{O}$ & -0.264 & -0.264 & -0.261 & -0.258 & -0.975 & 0.009 \\
$\mathrm{~F}$ & -0.326 & -0.326 & -0.322 & -0.322 & -1.166 & 0.008 \\
$\mathrm{Ne}$ & -0.383 & -0.383 & -0.376 & -0.390 & -1.374 & 0.005 \\
$\mathrm{Na}$ & -0.410 & -0.409 & -0.401 & -0.398 & -1.577 & 0.004 \\
$\mathrm{Mg}$ & -0.462 & -0.460 & -0.452 & -0.444 & -1.782 & 0.014 \\
$\mathrm{Al}$ & -0.498 & -0.497 & -0.491 & -0.479 & -1.987 & 0.028 \\
$\mathrm{Si}$ & -0.534 & -0.532 & -0.527 & -0.520 & -2.198 & 0.050 \\
$\mathrm{P}$ & -0.568 & -0.567 & -0.559 & -0.553 & -2.412 & 0.078 \\
$\mathrm{~S}$ & -0.636 & -0.635 & -0.629 & -0.634 & -2.631 & 0.110 \\
$\mathrm{Cl}$ & -0.696 & -0.695 & -0.689 & -0.714 & -2.855 & 0.149 \\
$\mathrm{Ar}$ & -0.752 & -0.751 & -0.744 & -0.787 & -3.084 & 0.193 \\
\hline \hline
\end{tabular}

${ }^{\mathrm{a} B L Y P}$ without the KLI-SIC.

${ }^{\mathrm{b}} \mathrm{BLYP}$ with the KLI-SIC.

${ }^{\mathrm{c}}$ Reference [14].

${ }^{\mathrm{d}}$ Reference [33].

sults shown in Tables I and II clearly indicate that the KLI SIC is an excellent procedure for improving the LSDA energy functional.

\section{B. Ionization potentials for neutral atoms with $Z \leqslant 18$}

The ionization potential (IP) is defined as the difference of the total energy of a parent neutral atom and its corresponding singly ionized atom. In the independent-particle approximation such as the KS approach, the energy of the highest occupied orbital can be also taken as the IP according to the work of Perdew et al. [27]. For the study of dynamical processes such as photoionization and multiphoton ionization, it is essential that the orbital energy be described correctly. In Table IV we list the calculated IPs of neutral atoms $(Z \leqslant 18)$ based on the energy of the highest occupied orbital for individual atom. Since both LSDA and BLYP energy functionals (without the KLI-SIC) do not have the correct long-range behavior, their IPs are significantly smaller than the experimental values by $40-50 \%$ with the LSDA results uniformly worse. With the implementation of the KLI-SIC, the ionization potentials of both the LSDA and BLYP are much improved. Except for a few cases, the BLYP ionization potentials are generally better than those of the LSDA. In a comparison with the IPs of Ref. [14], our BLYP-KLI-SIC values are comparable in accuracy, better in some cases, but worse in other cases. Finally, we note that our LSDA-KLI-SIC results coincide with the recent calculations by Chen et al. [25] using a similar procedure.

\section{Electron affinities}

Another more crucial test of the quality of energy functionals is the prediction of the IPs or the electron affinities of 
TABLE IV. Ionization potentials (in a.u.) of ground states of neutral atoms $(Z \leqslant 18)$ calculated from the highest occupied orbital energies by various exchange-correlation energy functionals.

\begin{tabular}{lccccccc}
\hline \hline & & \multicolumn{2}{c}{ Non-KLI-SIC } & \multicolumn{2}{c}{ KLI-SIC } & \multicolumn{2}{c}{ KLI } \\
Atom & HF $^{\mathrm{a}}$ & LSDA & BLYP & LSDA & BLYP & GG $^{\mathrm{b}}$ & Expt. $^{\mathrm{c}}$ \\
\hline $\mathrm{He}$ & 0.918 & 0.517 & 0.585 & 0.918 & 0.950 & 0.945 & 0.904 \\
$\mathrm{Li}$ & 0.196 & 0.100 & 0.111 & 0.196 & 0.194 & 0.200 & 0.198 \\
$\mathrm{Be}$ & 0.309 & 0.170 & 0.201 & 0.308 & 0.323 & 0.329 & 0.343 \\
$\mathrm{~B}$ & 0.310 & 0.120 & 0.143 & 0.290 & 0.304 & 0.328 & 0.305 \\
$\mathrm{C}$ & 0.433 & 0.196 & 0.218 & 0.412 & 0.422 & 0.448 & 0.414 \\
$\mathrm{~N}$ & 0.568 & 0.276 & 0.297 & 0.536 & 0.543 & 0.579 & 0.534 \\
$\mathrm{O}$ & 0.632 & 0.210 & 0.266 & 0.479 & 0.523 & 0.559 & 0.501 \\
$\mathrm{~F}$ & 0.730 & 0.326 & 0.377 & 0.645 & 0.680 & 0.714 & 0.640 \\
$\mathrm{Ne}$ & 0.850 & 0.443 & 0.492 & 0.808 & 0.837 & 0.884 & 0.793 \\
$\mathrm{Na}$ & 0.182 & 0.097 & 0.107 & 0.187 & 0.184 & 0.189 & 0.189 \\
$\mathrm{Mg}$ & 0.253 & 0.142 & 0.168 & 0.256 & 0.267 & 0.273 & 0.281 \\
$\mathrm{Al}$ & 0.210 & 0.086 & 0.102 & 0.192 & 0.198 & 0.222 & 0.217 \\
$\mathrm{Si}$ & 0.297 & 0.144 & 0.160 & 0.275 & 0.279 & 0.304 & 0.300 \\
$\mathrm{P}$ & 0.392 & 0.203 & 0.219 & 0.358 & 0.361 & 0.399 & 0.385 \\
$\mathrm{~S}$ & 0.437 & 0.174 & 0.219 & 0.344 & 0.375 & 0.404 & 0.381 \\
$\mathrm{Cl}$ & 0.506 & 0.254 & 0.295 & 0.447 & 0.472 & 0.506 & 0.477 \\
$\mathrm{Ar}$ & 0.591 & 0.334 & 0.373 & 0.549 & 0.571 & 0.619 & 0.579 \\
\hline \hline
\end{tabular}

${ }^{\mathrm{a}}$ Reference [30].

${ }^{\mathrm{b}}$ Reference [14].

${ }^{\mathrm{c}}$ Reference [34].

negative ions. It is known that within the standard $\mathrm{KS}$ scheme using local energy functionals (such as the LSDA and BLYP forms), the electrons are too weakly bound and there is no convergence for negative ions. With the use of the KLI-SIC procedure; however, we are able to calculate the electron affinities for most negative ions. The results are shown in Table V. Here we present the electron affinities obtained both from the energy of the highest occupied orbital [shown in column (a)] as well as from the energy difference between the negative ions and their corresponding neutral atoms [shown in column (b)]. For closed-shell negative ions, our results are in reasonable agreement with the experimental values. The predicted electron affinities for open-shell negative ions are less satisfactory as we use a spherical symmetrized density without polarization. One noticeable trend coming out from our KLI-SIC studies is that the two different estimations of the electron affinities [column (a) and column (b) in both the LSDA and BLYP with the KLI-SIC] merge to each other as the atomic number $Z$ increases, indicating that the Koopmans theorem is more valid for heavier atoms. However, the results of columns (a) and (b) are quite different for the KLI calculations in Ref. [14].

\section{TIME-DEPENDENT LINEAR DENSITY RESPONSE THEORY WITH OPTIMIZED EFFECTIVE POTENTIAL AND SELF-INTERACTION CORRECTION: APPLICATION TO PHOTOIONIZATION OF NEON ATOMS}

We now consider the extension of the OEP-KLI method with SIC procedure to the dynamical process of photoionization of rare gas atoms. In the context of DFT, there are two general approaches for the study of photoresponse; both are
TABLE V. Electron affinities (in a.u.) of negative ions $(Z \leqslant 18)$ calculated by various exchange-correlation energy functionals: (a) electron affinity calculated from the highest occupied orbital energy and (b) electron affinity calculated from the difference of the total energies between the negative ion and the corresponding neutral atom.

\begin{tabular}{lccccccc}
\hline \hline & \multicolumn{3}{c}{ LSDA-KLI-SIC } & \multicolumn{3}{c}{ BLYP-KLI-SIC } & \multicolumn{3}{c}{ GG $^{\mathrm{a}}$} \\
Ion & (a) & (b) & (a) & (b) & (a) & (b) & Expt. \\
\hline $\mathrm{H}^{-}$ & 0.046 & -0.012 & 0.063 & 0.019 & & & 0.028 \\
$\mathrm{Li}^{-}$ & 0.015 & -0.003 & 0.023 & 0.017 & 0.024 & 0.016 & 0.023 \\
$\mathrm{~B}^{-}$ & 0.021 & 0.013 & 0.025 & 0.017 & 0.033 & -0.002 & 0.010 \\
$\mathrm{C}^{-}$ & 0.064 & 0.049 & 0.068 & 0.051 & 0.083 & 0.028 & 0.046 \\
$\mathrm{O}^{-}$ & 0.064 & 0.026 & 0.096 & 0.053 & 0.110 & 0.017 & 0.054 \\
$\mathrm{~F}^{-}$ & 0.160 & 0.102 & 0.185 & 0.124 & 0.208 & 0.082 & 0.125 \\
$\mathrm{Na}^{-}$ & 0.014 & -0.001 & 0.022 & 0.018 & 0.022 & 0.015 & 0.020 \\
$\mathrm{Al}^{-}$ & 0.016 & 0.015 & 0.017 & 0.015 & 0.024 & 0.007 & 0.016 \\
$\mathrm{Si}^{-}$ & 0.047 & 0.048 & 0.049 & 0.047 & 0.065 & 0.040 & 0.051 \\
$\mathrm{P}^{-}$ & 0.001 & -0.001 & 0.037 & 0.029 & 0.048 & 0.022 & 0.027 \\
$\mathrm{~S}^{-}$ & 0.067 & 0.057 & 0.090 & 0.080 & 0.106 & 0.065 & 0.076 \\
$\mathrm{Cl}^{-}$ & 0.126 & 0.115 & 0.146 & 0.133 & 0.174 & 0.122 & 0.133 \\
\hline \hline
\end{tabular}

${ }^{\mathrm{a}}$ Reference [14].

${ }^{\mathrm{b}}$ Reference [34].

based on the lowest-order perturbation theory. The first approach is the direct extension of the Kohn-Sham independent-particle self-consistent-field (SCF) approach with the use of the LDA for the density functional [35]. In this approach [often referred to as the time-independent LDA (LSDA) method], the screening effect or the electron polarization effect induced by the external field is not considered. Another more rigorous approach, taking into account the time-dependent perturbation and the screening effects, is based on the time-dependent linear density response theory [35-37]. The LDA or LSDA is often used along with this approach. [This procedure is usually referred to as the timedependent LDA (LSDA) method.] Both time-dependent and time-independent LDA (LSDA) methods have been applied to the study of dynamical polarizability and photoionization cross sections of atoms and molecules [35]. The results of time-dependent LDA calculations are found to be generally more accurate than those of time-independent LDA calculations [35], indicating the importance of including the screening effects in the study of dynamical properties. However, since both the LDA (LSDA) and more accurate forms of density functionals such as the BLYP functional do not support the correct long-range Coulombic behavior as shown in Figs. 2(a) and 2(b), the electrons are too weakly bound. Previous time-dependent LDA studies $[9,35]$ of the photoionization of rare gas atoms, for example, did not exhibit the autoionizing resonances. Proper treatment of the long-range potential in DFT is essential to the study of Rydberg series, autoionizing resonances as well as the bound-free photoionization and multi-photon ionization processes. Recently, Stener et al. [38] have used the model potential of van Leeuwen and Baerends [39] for the study of photoionization of rare gas atoms. Since the Van Leeuwen-Baerends (VLB) potential implements the $(-1 / r)$ long-range Coulomb tail, they were able to study the singly excited autoionizing resonances. However, the VLB-type model potential is not ob- 
tained from the functional derivative of some xc-energy functional and thus is not completely compatible with DFT. In this section we present the extension of the OEP-KLI-SIC procedure to the time-dependent linear density response theory, allowing for a DFT approach for the study of photoionization and autoionizing resonances.

We start from the independent-particle approximation and consider the photoexcitation from an initial state $|i\rangle$ to a final state $|j\rangle$, where $|i\rangle$ and $|j\rangle$ are the solutions of the oneelectron OEP equations

$$
\left[-\frac{1}{2} \nabla^{2}+V^{\mathrm{OEP}}(\vec{r})\right] \phi_{i}(\vec{r})=\varepsilon_{i} \phi_{i}(\vec{r})
$$

Here $V^{\mathrm{OEP}}(\mathbf{r})$ is the optimized effective potential obtained by the KLI-SIC procedure outlined in Sec. II. For photoionization processes, the final states are unbound solutions of Eq. (27) with $\epsilon_{i}$ replaced by $\frac{1}{2} k^{2}$, where $k$ is the photoelectron momentum. The partial cross section is given by the usual expression

$$
\sigma_{i}(\omega)=\frac{2 \omega}{3} \frac{2 \pi^{2}}{c} n_{i} \sum_{j}\left(1-n_{j}\right)|\langle j|\mathbf{r}| i\rangle|^{2} \delta\left(\omega-\varepsilon_{j}+\varepsilon_{i}\right),
$$

where $n_{i}$ and $n_{j}$ are the occupation numbers of the initial and final states, respectively. The total cross section is obtained by summing over all the initial states,

$$
\sigma(\omega)=\sum_{i} \sigma_{i}(\omega)
$$

We next discuss the extension of the OEP-KLI-SIC method to the linear density response theory [9,36-38], which considers the effect of a weak time-dependent perturbation potential on the electron density. The frequency-dependent induced density $\delta \rho(\mathbf{r}, \omega)$ can be obtained by the Fourier transformation of the time-dependent field-induced density $\delta \rho(\mathbf{r}, t)$,

$$
\delta \rho(\mathbf{r}, \omega)=\frac{1}{2 \pi} \int_{-\infty}^{\infty} \delta \rho(\mathbf{r}, t) e^{i \omega t} d t
$$

The induced density is related to the external potential by the relationship

$$
\delta \rho(\mathbf{r}, \omega)=\int \chi\left(\mathbf{r}, \mathbf{r}^{\prime}, \omega\right) V^{\mathrm{ext}}\left(\mathbf{r}^{\prime}, \omega\right) d^{3} \mathbf{r}^{\prime},
$$

where $\chi\left(\mathbf{r}, \mathbf{r}^{\prime}, \omega\right)$ is the frequency-dependent susceptibility and

$$
V^{\operatorname{ext}}(\mathbf{r}, \omega)=z
$$

is the dipole external potential. The susceptibility can be determined by means of the first-order time-dependent perturbation theory [40] and expressed in terms of the eigenfunctions $\left\{\phi_{i}(\mathbf{r})\right\}$ and eigenvalues $\left\{\boldsymbol{\epsilon}_{i}\right\}$ of the solutions of the OEP equation (27)

$$
\chi_{\mathrm{OEP}}\left(\mathbf{r}, \mathbf{r}^{\prime}, \omega\right)=\sum_{i, j}\left(n_{i}-n_{j}\right) \frac{\phi_{i}^{*}(\mathbf{r}) \phi_{j}(\mathbf{r}) \phi_{i}\left(\mathbf{r}^{\prime}\right) \phi_{j}^{*}\left(\mathbf{r}^{\prime}\right)}{\omega-\left(\varepsilon_{j}-\varepsilon_{i}\right)+i \eta},
$$

where $i \eta$ is an imaginary infinitesimal used to ensure the outgoing wave boundary conditions. The summation over $i$ and $j$ runs over all the bound and continuum states. Within the SCF approximation, we can replace Eq. (31) with

$$
\delta \rho(\mathbf{r}, \omega)=\int \chi_{\mathrm{OEP}}\left(\mathbf{r}, \mathbf{r}^{\prime}, \omega\right) V^{\mathrm{SCF}}\left(\mathbf{r}^{\prime}, \omega\right) d^{3} \mathbf{r}^{\prime}
$$

where

$$
\begin{aligned}
V^{\mathrm{SCF}}(\mathbf{r}, \omega)= & V^{\operatorname{ext}}(\mathbf{r}, \omega)+\int \frac{\delta \rho\left(\mathbf{r}^{\prime}, \omega\right)}{\left|\mathbf{r}-\mathbf{r}^{\prime}\right|} d^{3} \mathbf{r}^{\prime} \\
& +\left.\frac{\partial V_{\mathrm{xc}}(\mathbf{r})}{\partial \rho(\mathbf{r})}\right|_{\rho_{0}(\mathbf{r})} \cdot \delta \rho(\mathbf{r}, \omega)
\end{aligned}
$$

Here $\rho_{0}(\mathbf{r})$ is the ground-state electron density, for example, $V_{\mathrm{xc}}=-(6 / \pi)^{1 / 3} \rho^{1 / 3}(\mathbf{r})$, for the LSDA. The normal procedure is to solve Eqs. (34) and (35) iteratively until convergence is reached. However, an alternative and simpler procedure can be obtained by substituting Eq. (34) into Eq. (35) to get

$$
V^{\mathrm{SCF}}(\mathbf{r}, \omega)=V^{\mathrm{ext}}(\mathbf{r}, \omega)+\int K\left(\mathbf{r}, \mathbf{r}^{\prime}, \omega\right) V^{\mathrm{SCF}}\left(\mathbf{r}^{\prime}, \omega\right) d^{3} \mathbf{r}^{\prime},
$$

with

$$
\begin{aligned}
K\left(\mathbf{r}, \mathbf{r}^{\prime}, \omega\right)= & \int \frac{\chi_{\mathrm{OEP}}\left(\mathbf{r}^{\prime}, \mathbf{r}^{\prime \prime}, \omega\right)}{\left|\mathbf{r}-\mathbf{r}^{\prime \prime}\right|} d^{3} \mathbf{r}^{\prime \prime} \\
& +\left.\frac{\partial V_{\mathrm{xc}}(\mathbf{r})}{\partial \rho(\vec{r})}\right|_{\rho_{0}(\mathbf{r})} \cdot \chi_{\mathrm{OEP}}\left(\mathbf{r}, \mathbf{r}^{\prime}, \omega\right) .
\end{aligned}
$$

The integral equation (36) can now be recasted into the linear equation form

$$
\int\left[\delta\left(\mathbf{r}-\mathbf{r}^{\prime}\right)-K\left(\mathbf{r}, \mathbf{r}^{\prime}, \omega\right)\right] V^{\mathrm{SCF}}\left(\mathbf{r}^{\prime}, \omega\right)=V^{\operatorname{ext}}(\mathbf{r}, \omega),
$$

from which $V^{\mathrm{SCF}}(\mathbf{r}, \omega)$ can be readily solved by discretizing the $\mathbf{r}$ space. Substituting the results of $V^{\mathrm{SCF}}$ into Eq. (34), we obtain the desired induced density $\delta \rho(\mathbf{r}, \omega)$. Finally, the cross section can be obtained by the well-known relationship

$$
\sigma(\omega)=\frac{4 \pi \omega}{c} \operatorname{Im}[\alpha(\omega)]
$$

where $\alpha(\omega)$ is the dynamical polarizability given by

$$
\alpha(\omega)=-\iint V^{\mathrm{ext}}(\mathbf{r}, \omega) \chi_{\mathrm{OEP}}\left(\mathbf{r}, \mathbf{r}^{\prime}, \omega\right) \cdot V^{\mathrm{SCF}}\left(\mathbf{r}^{\prime}, \omega\right) d^{3} \mathbf{r}
$$


The key issue here is how to calculate the susceptibility based on the OEP. Since the susceptibility can be written as a summation over all orbitals, we can calculate the contributions of the susceptibility by the Green's-function method as discussed in [9]. First we rewrite Eq. (33) as

$$
\begin{aligned}
\chi_{\mathrm{OEP}}\left(\mathbf{r}, \mathbf{r}^{\prime}, \omega\right)= & \sum_{i, j} n_{i} \frac{\phi_{i}^{*}(\mathbf{r}) \phi_{j}(\mathbf{r}) \phi_{i}\left(\mathbf{r}^{\prime}\right) \phi_{j}^{*}\left(\mathbf{r}^{\prime}\right)}{\omega-\left(\varepsilon_{j}-\varepsilon_{i}\right)+i \eta} \\
& -\sum_{i, j} n_{j} \frac{\phi_{i}^{*}(\mathbf{r}) \phi_{j}(\mathbf{r}) \phi_{i}\left(\mathbf{r}^{\prime}\right) \phi_{j}^{*}\left(\mathbf{r}^{\prime}\right)}{\omega-\left(\varepsilon_{j}-\varepsilon_{i}\right)+i \eta} \\
= & \sum_{i} n_{i} \phi_{i}^{*}(\mathbf{r}) \phi_{i}\left(\mathbf{r}^{\prime}\right) \sum_{j} \frac{\phi_{j}(\mathbf{r}) \phi_{j}^{*}\left(\mathbf{r}^{\prime}\right)}{\omega-\left(\varepsilon_{j}-\varepsilon_{i}\right)+i \eta} \\
& +\sum_{i} n_{i} \phi_{i}(\mathbf{r}) \phi_{i}^{*}\left(\mathbf{r}^{\prime}\right) \\
& \times \sum_{j} \frac{\phi_{j}^{*}(\mathbf{r}) \phi_{j}\left(\mathbf{r}^{\prime}\right)}{-\omega-\left(\varepsilon_{j}-\varepsilon_{i}\right)-i \eta} \\
\equiv & \sum_{i} n_{i} \phi_{i}^{*}(\mathbf{r}) \phi_{i}\left(\mathbf{r}^{\prime}\right) G\left(\mathbf{r}, \mathbf{r}^{\prime} ; \omega+\varepsilon_{i}+i \eta\right) \\
& +\sum_{i} n_{i} \phi_{i}(\mathbf{r}) \phi_{i}^{*}\left(\mathbf{r}^{\prime}\right) G^{*}\left(\mathbf{r}, \mathbf{r}^{\prime} ; \varepsilon_{i}-\omega+i \eta\right)
\end{aligned}
$$

and then expand the Green's function in terms of spherical harmonics

$$
G\left(\mathbf{r}, \mathbf{r}^{\prime} ; E\right)=\sum_{L} Y_{L}^{*}(\hat{\mathbf{r}}) G_{L}\left(r, r^{\prime} ; E\right) Y_{L}\left(\hat{\mathbf{r}}^{\prime}\right)
$$

where $L$ is a compact notation for the angular momentum quantum numbers $l, m$. The radial Greens's function $G_{L}\left(r, r^{\prime} ; E\right)$ can be determined by

$$
G_{L}\left(r, r^{\prime} ; E\right)=\frac{j_{l}\left(r_{<}\right) h_{l}\left(r_{>}\right)}{W\left[j_{l}, h_{l}\right]},
$$

where $r_{<}\left(r_{>}\right)$refers to the smaller (larger) distance of $r$ and $r^{\prime} \cdot j_{l}(r)$ is the partial wave solution of Eq. (27) with energy $E$ and satisfies the proper boundary condition at the origin. Similarly, $h_{l}(r)$ is the partial wave solution of Eq. (27) with energy $E$ and satisfies the outgoing boundary condition as $r \rightarrow \infty$ for $E>0$ or the decaying behavior solution as $r \rightarrow \infty$ for $E \leqslant 0 . W\left[j_{l}, h_{l}\right]$ is the Wronskian of $j_{l}(r)$ and $h_{l}(r)$. With the calculated Green's functions, we can construct the susceptibility from Eq. (41). Once the susceptibility is determined, the self-consistent field $V^{\mathrm{SCF}}$ is obtained by the solution of Eq. (38) and the cross section can be calculated by Eqs. (39) and (40). The linear response method is usually referred as a time-dependent technique. If we use $V^{\text {ext }}$ instead of $V^{\mathrm{SCF}}$ in Eq. (40) we reproduce the crosssection expression for the independent-particle approximation. The independent-particle approximation [Eq. (28)] will be referred to as the time-independent method since it does not take into account time-dependent field-induced density.
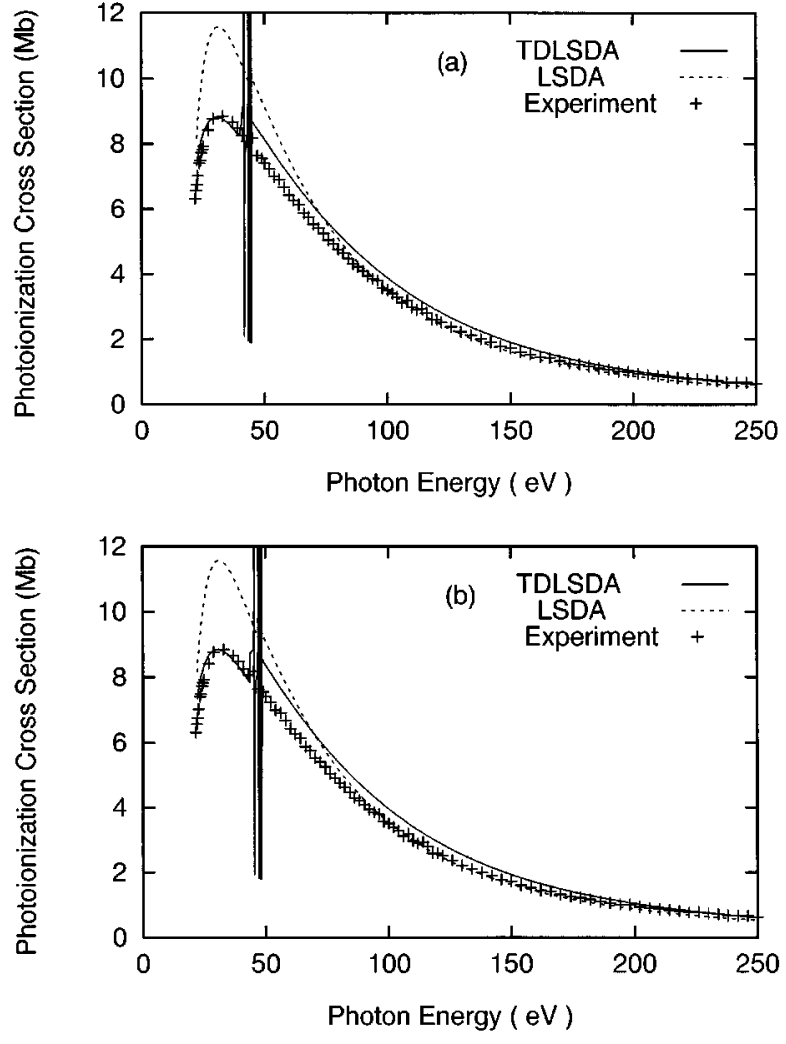

FIG. 3. Total photoionization cross sections of time-independent and time-dependent calculations with the LSDA-KLI-SIC potential: (a) present $a b$ initio results and (b) present results calculated with the $2 s$ orbital energy replaced by the experimental value.

[To test the numerical accuracy of the Green's-function method, we have calculated the photoionization cross sections from Eqs. (39) and (40) using $V^{\text {ext }}$ instead of $V^{\mathrm{SCF}}$ and compared the results with those calculated directly from Eq. (28). The two sets of results are in agreement with each other. In the following discussions, all the time-independent cross sections were obtained from Eqs. (39) and (40) using $V^{\mathrm{ext}}$ instead of $V^{\mathrm{SCF}}$.]

To illustrate the usefulness of the optimized effective potential method with the KLI-SIC for dynamical processes, we have performed the calculation of photoionization cross sections of the $\mathrm{Ne}$ atom using both time-independent and time-dependent methods. Figure 3 shows the results of timeindependent LSDA (denoted LSDA in the figure) and timedependent LSDA (denoted TDLSDA in the figure) calculations, both with the KLI-SIC. Although our calculated $2 s$ orbital energy with the KLI-SIC has improved the LSDA value from 1.266 a.u. to 1.645 a.u., there is still some discrepancy from the experimental value 1.782 a.u. [41]. [The calculated $2 p$ (OEP-KLI-SIC) orbital energy 0.808 a.u. is much closer to the experimental value 0.793 a.u.] To see the influence of such a $2 s$ energy shift to the photoionization cross sections and the resonance profiles, we presented in Figs. 3(a) and 3(b) two sets of data, one from the ab initio OEP-KLI-SIC calculations [Fig. 3(a)] and the other from using the experimental value of the $2 s$ orbital energy in the Green's-function calculations [Fig. 3(b)]. Also shown in both Figs. 3(a) and 3(b) are the recent experimental results [42] for comparison. 


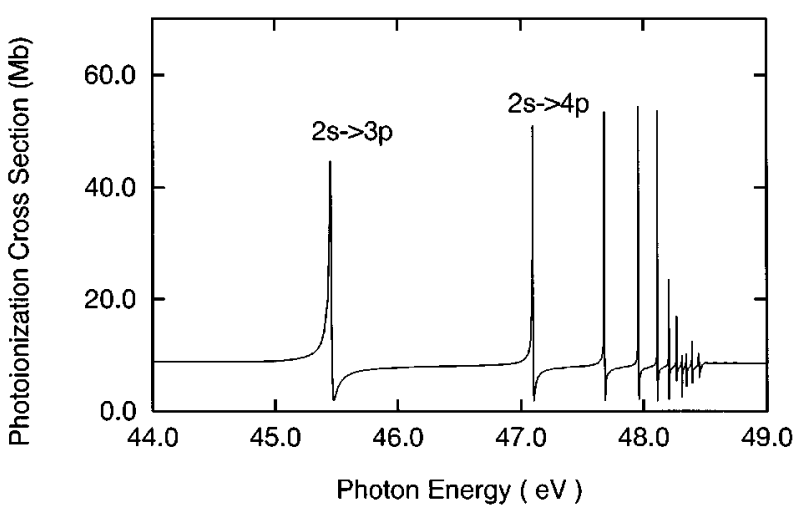

FIG. 4. Photoionization cross sections near the $2 s \rightarrow n p$ resonant transitions, showing the autoionizing resonance profiles. The results are obtained by the TDLSDA with the KLI-SIC potential. The experimental value of the $2 s$ orbital energy is used in the calculation.

Figure 3(a) shows that the total cross sections of the timeindependent LSDA calculations are substantially larger than the experimental values in the broad peak region and a small bump appears near the $2 s$ ionization threshold due to the contribution from the photoionization of the $2 s$ level. Above the $2 s$ ionization threshold, the cross sections decrease monotonically. The TDLSDA cross sections show significant improvement and close agreement with the experimental data in the broad peak region followed by a series of sharp resonances due to $2 s \rightarrow n p$ transitions near the $2 s$ threshold. Above the $2 s$ ionization threshold, both the LSDA and TDLSDA results have some small discrepancy with the experimental data. However, the LSDA and TDLSDA results merge to each other in the higher-energy regime and follow closely the experimental values. Figure 3(b) shows the effect of implementing the experimental $2 s$ orbital energy in the calculations. Apart from the slight shift of the resonance positions, the overall photoionization cross-section profiles are not significantly affected.

In Fig. 4 we show the details of the photoionization cross sections in the $2 s \rightarrow n p$ resonance region with the $2 s$ orbital energy corrected by the experimental value. To determine the autoionization widths, we fit the resonance line shapes to the Fano profile [43]

$$
\sigma(\omega)=\sigma_{0}\left(\rho^{2} \frac{(q+x)^{2}}{1+x^{2}}-\rho^{2}+1\right)
$$

with

$$
x=\frac{\omega-E_{r}}{\frac{1}{2} \Gamma} .
$$

Here $\sigma_{0}$ is the cross section, $q$ is a line profile index, $E_{r}$ is the resonance position, $\Gamma$ is the resonance half-width, and $\rho^{2}$ is the correlation coefficient, which gives the proportion of the continuum that interacts with the autoionizing states.

The fitted resonance parameters are listed in Table VI. Two sets of our results are presented: TDLSDA ${ }^{\text {a }}$ refers to the $a b$ initio KLI-SIC calculations using the TDLSDA method
TABLE VI. Comparison of line profile parameters of autoionizing resonances for $\mathrm{Ne}$ atom.

\begin{tabular}{lccccc}
\hline \hline & $\sigma_{0}(\mathrm{Mb})$ & $\Gamma(\mathrm{meV})$ & $E_{r}(\mathrm{eV})$ & $q$ & $\rho^{2}$ \\
\hline & & \multicolumn{5}{c}{$2 s \rightarrow 3 p$} \\
Ref. [38] & 8.18 & 13.90 & 46.253 & -3.69 & 0.514 \\
TDLSDA $^{\mathrm{a}}$ & 8.37 & 16.49 & 41.714 & -2.64 & 0.749 \\
TDLSDA $^{\mathrm{b}}$ & 8.28 & 18.04 & 45.453 & -2.40 & 0.764 \\
Expt. $^{\mathrm{c}}$ & $8.6 \pm 0.6$ & $13 \pm 2$ & 45.546 & $-1.6 \pm 0.3$ & $0.7 \pm 0.07$ \\
CI $^{\mathrm{d}}$ & & 11.7 & & -1.61 & 0.76 \\
& & & & & \\
& & & $2 s \rightarrow 4 p$ & & \\
Ref. [38] & 7.98 & 3.86 & 47.397 & -3.95 & 0.505 \\
TDLSDA $^{\mathrm{a}}$ & 8.20 & 4.73 & 43.361 & -2.82 & 0.765 \\
TDLSDA $^{\mathrm{b}}$ & 8.06 & 5.14 & 47.098 & -2.62 & 0.783 \\
Expt. $^{\mathrm{c}}$ & $8.0 \pm 0.6$ & $4.5 \pm 1.5$ & 47.121 & $-1.6 \pm 0.3$ & $0.7 \pm 0.07$ \\
CI $^{\mathrm{d}}$ & & 3.8 & & -1.75 & 0.76 \\
& & & & & \\
& & & $2 s \rightarrow 5 p$ & & \\
Ref. [38] & 7.91 & 1.62 & 47.814 & -4.05 & 0.502 \\
TDLSDA $^{\mathrm{a}}$ & 7.94 & 2.02 & 43.361 & -2.96 & 0.765 \\
TDLSDA $^{\mathrm{b}}$ & 7.91 & 2.20 & 47.683 & -2.72 & 0.783 \\
Expt. $^{\mathrm{c}}$ & $8.2 \pm 0.6$ & $2 \pm 1$ & 47.692 & $-1.6 \pm 0.5$ & $0.7 \pm 0.14$ \\
\hline \hline
\end{tabular}

${ }^{\mathrm{a}}$ Present $a b$ initio TDLSDA results.

${ }^{\mathrm{b}}$ Present TDLSDA results with the $2 s$ orbital energy replaced by the experimental value.

${ }^{\mathrm{c}}$ Reference [44].

${ }^{\mathrm{d}}$ Reference [45].

and TDLSDA ${ }^{\mathrm{b}}$ refers to the same calculation except that the experimental value is used for the $2 s$ orbital energy. Also shown in Table VI are the experimental values [44], the configuration-interaction (CI) ( $R$-matrix) results [45], and the VLB model potential results [38] for the comparison. Except for the linewidth of $2 s \rightarrow 3 p$ transition, the TDLSDA-KLI-SIC results are in closer agreement with the experimental data [44] than those of model potential results [38]. The $q$ values of the present TDLSDA and model potential [38] calculations are somewhat larger than the experimental and CI values. With the $2 s$ orbital energy replaced by the experimental value, our predicted resonance positions $E_{r}$ for $2 s \rightarrow 3 p, 4 p$, and $5 p$ are in very close agreement with the experimental values, indicating that the excited-state properties are better described by the present KLI-SIC potential than by the VLB model potential [38].

\section{CONCLUSION}

In conclusion, we have presented in this paper a DFT procedure for improving the accuracy of the conventional density functionals for the treatment of ionization potentials, excited-state properties, and dynamical processes. Both the short- and long-range effective one-particle potentials are properly treated by the present OEP-KLI-SIC method. Further, only the local one-particle potential is used in this method, leading to a computationally efficient technique for DFT calculations of both ground and excited states. The usefulness and accuracy of the proposed KLI-SIC procedure are 
demonstrated by a study of both the static properties (total and ionization energies) of atoms $(Z \leqslant 18)$ and the photoionization process of $\mathrm{Ne}$ involving singly excited autoionizing resonances. We are currently extending the OEP-KLI-SIC method to the time-dependent processes of multiphoton and above-threshold ionization of many-electron systems in intense short-pulse laser fields.

\section{ACKNOWLEDGMENTS}

This work is partially supported by National Science Foundation under Contract No. PHY-95-12100. We are grateful to Dr. Weitao Yang for many instructive discussions of the density-functional theory at the beginning stage of this study and the comments on the manuscript.
[1] P. Hohenberg and W. Kohn, Phys. Rev. 136, B864 (1964).

[2] W. Kohn and L. J. Sham, Phys. Rev. 140, A1133 (1965).

[3] R. G. Parr and W. T. Yang, Density-Functional Theory of Atoms and Molecules (Oxford University Press, New York, 1989).

[4] R. M. Dreizler and E. K. U. Gross, Density Functional Theory, An Approach to the Quantum Many-Body Problem (Springer, Berlin, 1990).

[5] Density Functional Theory, Vol. 337 of NATO Advanced Study Institute, Series B: Physics, edited by E. K. U. Gross and R. M. Dreizler (Plenum, New York, 1995).

[6] N. H. March, Electron Density Theory of Atoms and Molecules (Academic, San Diego, 1992).

[7] Density Functional Methods in Chemistry, edited by J. K. Labanowski and J. W. Andzelm (Springer, Berlin, 1991).

[8] S. J. Vosko, L. Wilk, and M. Nusair, Can. J. Phys. 58, 1200 (1980).

[9] A. Zangwill and P. Stoven, Phys. Rev. A 21, 1561 (1980).

[10] A. D. Becke, Phys. Rev. A 38, 3098 (1988).

[11] C. Lee, W. Yang, and R. G. Parr, Phys. Rev. B 37, 785 (1988).

[12] J. P. Perdew and Y. Wang, Phys. Rev. B 33, 8800 (1986).

[13] Q. Zhao and R. G. Parr, Phys. Rev. A 46, R5320 (1992).

[14] T. Grabo and E. K. U. Gross, Chem. Phys. Lett. 240, 141 (1995).

[15] J. P. Perdew and A. Zunger, Phys. Rev. B 23, 5048 (1981).

[16] R. T. Sharp and G. K. Horton, Phys. Rev. 90, 317 (1953).

[17] J. D. Talman and W. F. Shadwick, Phys. Rev. A 14, 36 (1976).

[18] J. B. Krieger, Y. Li, and G. J. Iafrate, in Density Functional Theory, edited by E. K. U. Gross and R. M. Dreizler (Plenum, New York, 1994) p. 191.

[19] J. B. Krieger, Y. Li, and G. J. Iafrate, Phys. Rev. A 46, 5453 (1992).

[20] J. B. Krieger, Y. Li, and G. J. Iafrate, Phys. Lett. A 146, 256 (1990).

[21] J. B. Krieger, Y. Li, and G. J. Iafrate, Phys. Rev. A 45, 101 (1992).

[22] Y. Li, J. B. Krieger, and G. J. Iafrate, Phys. Rev. A 47, 165 (1993).
[23] X. M. Tong and Shih-I Chu (unpublished).

[24] J. B. Krieger, J. Chen, Y. Li, and G. J. Iafrate, Int. J. Quantum Chem. Symp. 29, 79 (1995).

[25] J. Chen, J. B. Krieger, Y. Li, and G. J. Iafrate, Phys. Rev. A 54, 3939 (1996).

[26] M. R. Norman and D. D. Koelling, Phys. Rev. B 30, 5530 (1984).

[27] J. P. Perdew, R. G. Parr, M. Levy, and J. L. Balduz, Jr., Phys. Rev. Lett. 49, 1691 (1981).

[28] E. R. Davidson, S. A. Hagstrom, S. J. Chakravorty, V. M. Umar, and C. F. Fischer, Phys. Rev. A 44, 7071 (1991).

[29] M. T. Carrol, R. F. W. Bader, and S. H. Vosko, J. Phys. B 20, 3599 (1987).

[30] C. F. Fischer, The Hartree-Fock Method for Atoms, A Numerical Approach (Wiley, New York, 1977).

[31] R. Colle and D. Salvetti, Theor. Chim. Acta 37, 329 (1975).

[32] R. Colle and D. Salvetti, Theor. Chim. Acta 53, 55 (1979).

[33] A. D. Becke, J. Chem. Phys. 96, 2155 (1992).

[34] A. A. Radzig and B. M. Smirnov, Reference Data on Atoms and Molecules (Springer, Berlin, 1985).

[35] G. D. Mahan and K. R. Subbaswamy, Local Density Theory of Polarizability (Plenum, New York, 1990), and references therein.

[36] E. Runge and E. K. U. Gross, Phys. Rev. Lett. 52, 997 (1984).

[37] E. K. U. Gross and W. Kohn, Phys. Rev. Lett. 55, 2850 (1985).

[38] M. Stener, P. Decleva, and A. Lisini, J. Phys. B 28, 4973 (1995).

[39] R. van Leeuwen and E. J. Baerends, Phys. Rev. A 49, 2421 (1994).

[40] A. L. Fetter and J. D. Walecka, Quantum Theory of ManyBody Systems (McGraw-Hill, New York, 1971).

[41] K. D. Sevier, At. Data Nucl. Data Tables 24, 327 (1979).

[42] W. F. Chan, G. Cooper, X. Guo, and C. E. Brion, Phys. Rev. A 45, 1420 (1992).

[43] U. Fano and J. W. Cooper, Phys. Rev. 137, A1364 (1965).

[44] K. Codling, R. P. Madden, and D. L. Ederer, Phys. Rev. 155, 26 (1967).

[45] P. G. Burke and K. T. Taylor, J. Phys. B 8, 2620 (1975). 